\title{
Archéopages
}

Archéopages

Archéologie et société

Hors-série 3 | 2012

Nouveaux champs de la recherche archéologique

\section{Des assemblages céramiques et lithiques « jalons » pour le Néolithique final en Auvergne : le site de Tremonteix}

\section{Sylvie Saintot}

\section{(2) OpenEdition}

Journals

Édition électronique

URL : https://journals.openedition.org/archeopages/696

DOI : 10.4000/archeopages.696

ISSN : 2269-9872

Éditeur

INRAP - Institut national de recherches archéologiques préventives

Édition imprimée

Date de publication : 1 janvier 2012

Pagination : 114-116

ISSN : 1622-8545

\section{Référence électronique}

Sylvie Saintot, «Des assemblages céramiques et lithiques « jalons » pour le Néolithique final en Auvergne : le site de Tremonteix », Archéopages [En ligne], Hors-série 3 | 2012, mis en ligne le 01 janvier 2012, consulté le 24 février 2023. URL : http://journals.openedition.org/archeopages/696 ; DOI : https://doi.org/10.4000/archeopages.696 


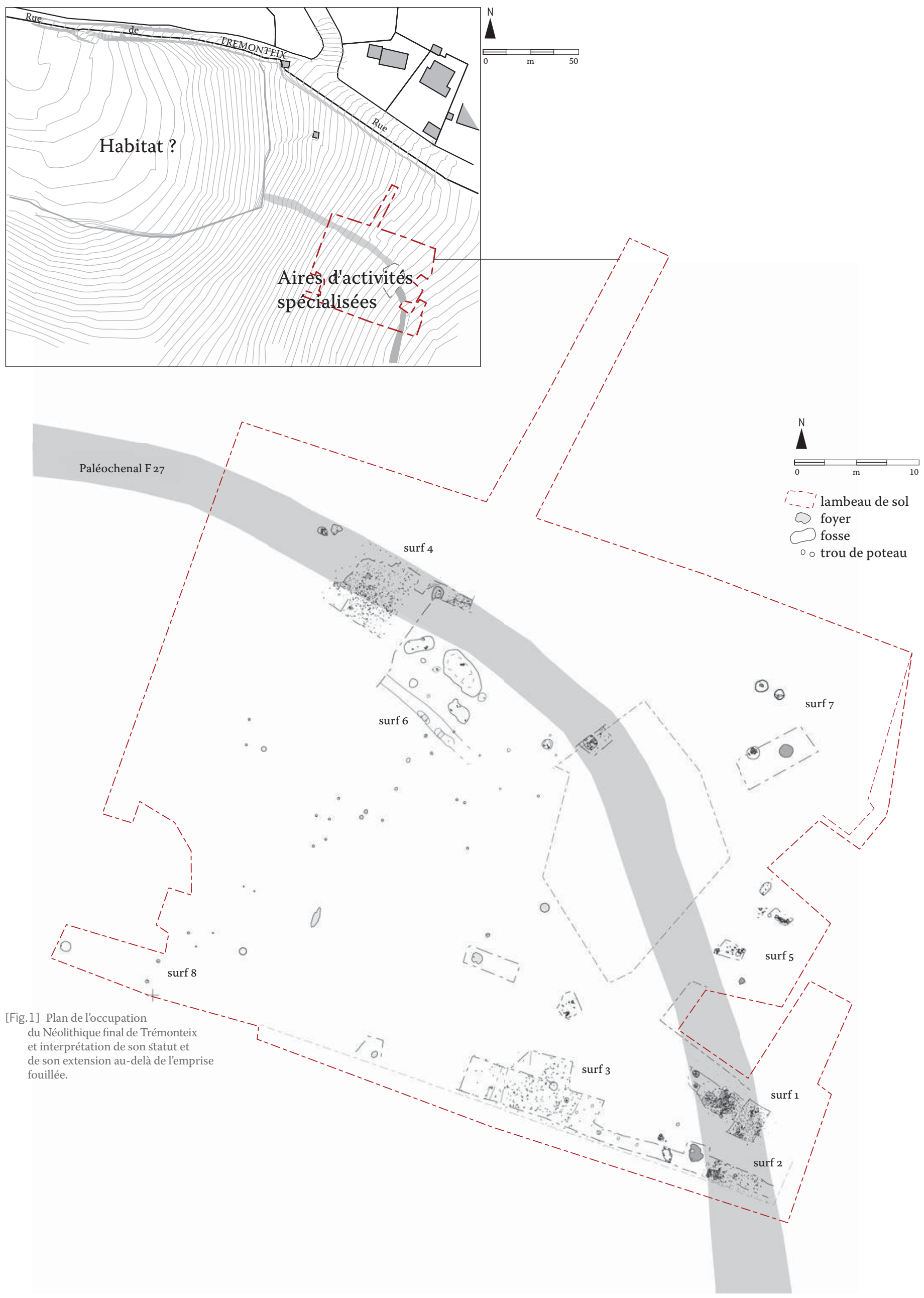




\section{Des assemblages céramiques et lithiques «jalons » pour le Néolithique final en Auvergne: le site de Tremonteix}

Sylvie Saintot

Inrap

$\mathbf{P}$ lusieurs occupations pré- et protohistoriques ont été découvertes dans ce secteur nord de Clermont-Ferrand, au pied des Côtes de Clermont. Si les indices de site de l'âge du Bronze se limitent à quelques tessons épars, attribués à la période du Campaniforme/Bronze ancien et à celle du Bronze final, ce n'est pas le cas de l'occupation du Néolithique final qui est majoritairement représentée à Trémonteix.

L'occupation néolithique qui s'étend sans doute au-delà [Fig.1], se singularise par de nombreux aménagements de l'emprise «en terrasse», «surcreusés » dans le lit d'un paléo-chenal et le long de sa rive droite. Plusieurs lambeaux de sols structurés, qui correspondent probablement à des aires d'activités collectives spécialisées, ont été mis en évidence dans ce paléo-chenal. De nombreuses chapes foyères, associées à des calages, sont aménagées dans ces horizons charbonneux et organiques, et plusieurs trous de poteaux et fosses-dépotoirs se répartissent le long de la bordure droite du paléo-chenal. Aménagées dans un secteur atteignant 10\% de pente, par endroit, ces aires d'activités se situent non loin de l'habitat, car les fosses et les lambeaux de sols ont livré de la céramique au répertoire typologique varié, du matériel lithique taillé abondant, de nombreux macro-restes, dont des graines de céréales rares dans ces contextes, de la faune majoritairement domestique, et de l'outillage en os et en bois de cerf. Globalement, le fonds commun des mobiliers s'accorde parfaitement avec celui des groupes du Néolithique final du Nord-Ouest de la France [Fig.2]. Ainsi, le répertoire abondant et varié de la céramique non décorée dénombre, entre autres, de nombreuses jarres à languettes à fond plat, plus rarement à cordon, des pots cylindriques, des bouteilles à col haut et à panse biconique, de nombreuses cuillères et louches, et quelques fusaïoles. Comme pour la céramique, le matériel lithique est assez diversifié. Il est représenté par de nombreux éclats et produits de mise en forme de livres de beurre en silex pressignien et par plusieurs outils. Parmi ceux-ci, outre les poignards, les pièces esquillées et les grattoirs, le taux élevé de microdenticulés $(65 \%)$ et la fragmentation intentionnelle des silex pressigniens, quels que soient les supports, sont ici tout à fait particuliers. La triade bœuf/porc/caprin s'accompagne, en moindres proportions, de cerf et de sanglier, alors que les industries osseuses sont représentées par des pointes et des poinçons, un racloir sur omoplate de bœuf et une hache-marteau en bois de cerf. Ces mobiliers du quotidien, particulièrement homogènes, sont les témoins d'une occupation relativement courte au cours du Néolithique final, même si les datations au carbone 14 réalisées sur os, calent le site entre 2500 et 2300 av. notre ère.
Lintérêt majeur du site néolithique de Trémonteix, repose avant tout sur l'abondance et la diversité du matériel céramique et lithique qui en font des assemblages « jalons » pour le Néolithique final auvergnat, le contexte chrono-culturel étant assez mal connu dans le Massif-Central au cours du troisième millénaire. En effet, à l'exception d'éléments isolés, les corpus de comparaison proviennent, pour l'essentiel, de deux sites auvergnats aux contextes distincts. Il s'agit d'une fosse unique, découverte à La Croix Saint-Roch, au Crest dan le Puy-de-Dôme (Hénon, 2006) et de l'habitat du Bournadiau à Quinssaines, situé au nord-ouest de l'Allier (Saintot, Linton, 2011), mais aucun de ces deux sites n'a livré d'assemblages fauniques, lithiques et surtout céramiques aussi conséquents que ceux qui ont été recueillis sur le site clermontois. Le site de Trémonteix est actuellement en cours d'étude et plusieurs questions se posent dès à présent. Quelle est la part réelle des composantes culturelles locales au sein des assemblages de mobilier, dans un contexte chronoculturel où les traditions extérieures prévalent $a$ priori ? Pourquoi y a-t-il une aussi forte proportion en silex pressigniens, acheminés sur des distances avoisinant les $300 \mathrm{~km}$ depuis leurs sources d'exploitation, et pourquoi démultiplier les supports en les sectionnant alors que le silex local est disponible à moins d'une vingtaine de kilomètres du site ? On s'interrogera enfin sur l'association cuillères/microdenticulés/ céréales, car ces trois éléments sont probablement concomitants et reflètent sans doute une activité économique vivrière et/ou artisanale importante au sein de la communauté de Trémonteix, comme sur d'autres sites du Nord de la France si l'on ne tient compte que des microdenticulés et des carporestes (Martial et al., 2011).

Outre la découverte de l'occupation même, les principales composantes des assemblages de mobilier nous renseignent en particulier sur les échanges économiques et sociaux entre les communautés du Nord-Ouest (Gord et Artenac en particulier), et celles du Centre de la France, au cœur du Massif-Central au cours du troisième millénaire ; la présence de groupes migrant du nord-ouest à Trémonteix n'est d'ailleurs pas à écarter.

CARLier M., FosSURIER C. (COLlab.), MENNESSIER-JOUANNET C. (COllab.), KildeA F. (COLLAB.), WitTMANN A. (COLlAB.), 2009: Clermont-Ferrand «ZAC de Trémonteix, tranche 1 » (Puy-de-Dôme), Rapport final d'opération de diagnostic, Inrap, Direction interrégionale Rhône-Alpes-Auvergne, 200 p., 71 fig.

Henon Ph., 2006 : « Le Crest (Puy-de-Dôme) La Croix Saint-Roch », Bilan scientifique de la région d'Auvergne 2006, SRA, Paris, Ministère de la Culture et de la communication, p. 106-107. martial E., Cayol N., Hamon C., Maigrot Y., Medard F., Monchablon C., 2011 : «Production et fonction des outillages au Néolithique final dans la vallée de la Deûle (Nord-Pas-de-Calais, France) », RAP, nº spécial, 28, p. 365-389.

Saintot S., Linton J., Baudais D. (Collab.), Combes P. (Collab.), Pelletier D. (Collab.), Weisser S. (Collab.), 2011 : « L'habitat du Néolithique final de Quinssaines, Le Bournadiau : approche spatiale et fonctionnelle d'un site au Nord-Ouest de l'Allier ", BSPF, T. $108, n^{\circ} 4$, p. 697-730.

.



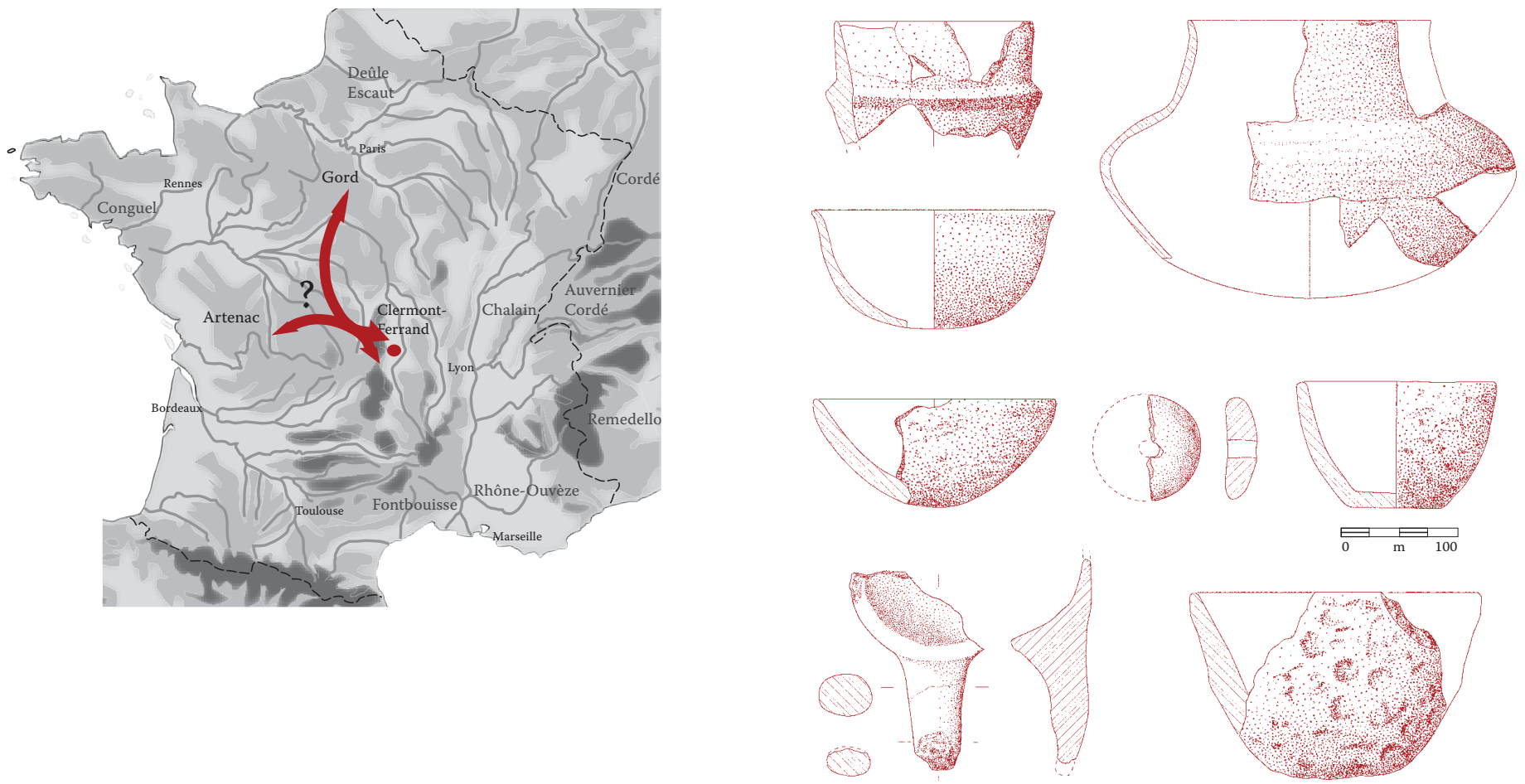

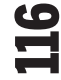
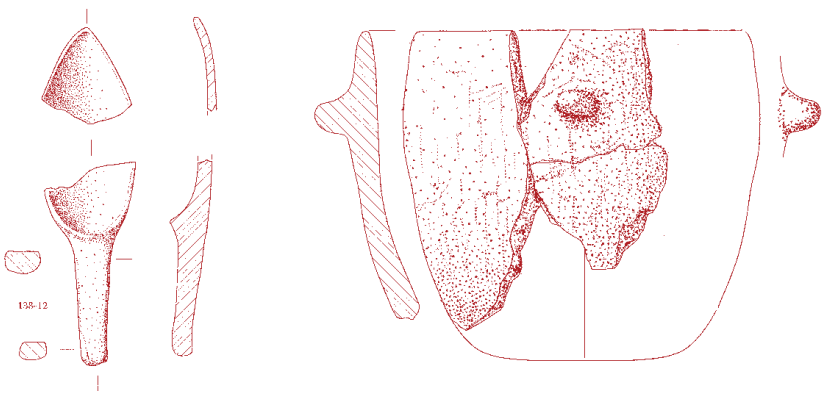

[Fig.2] Le site de Trémonteix dans son contexte chrono-culturel entre

2500 et 2300 av. notre ère et

échantillonnages des assemblages

céramiques et lithiques.
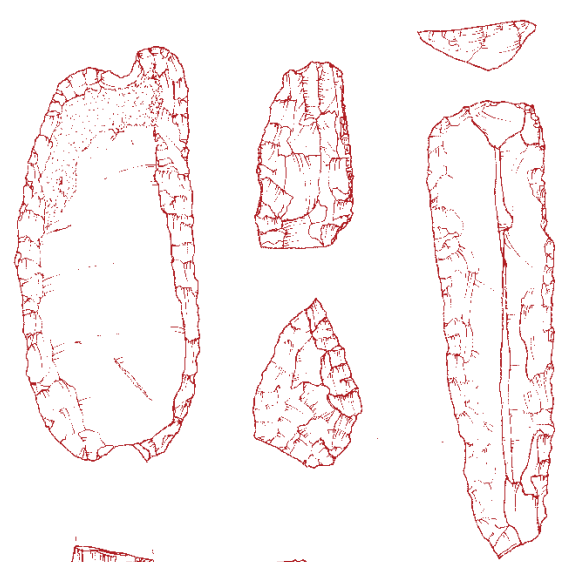

(E)
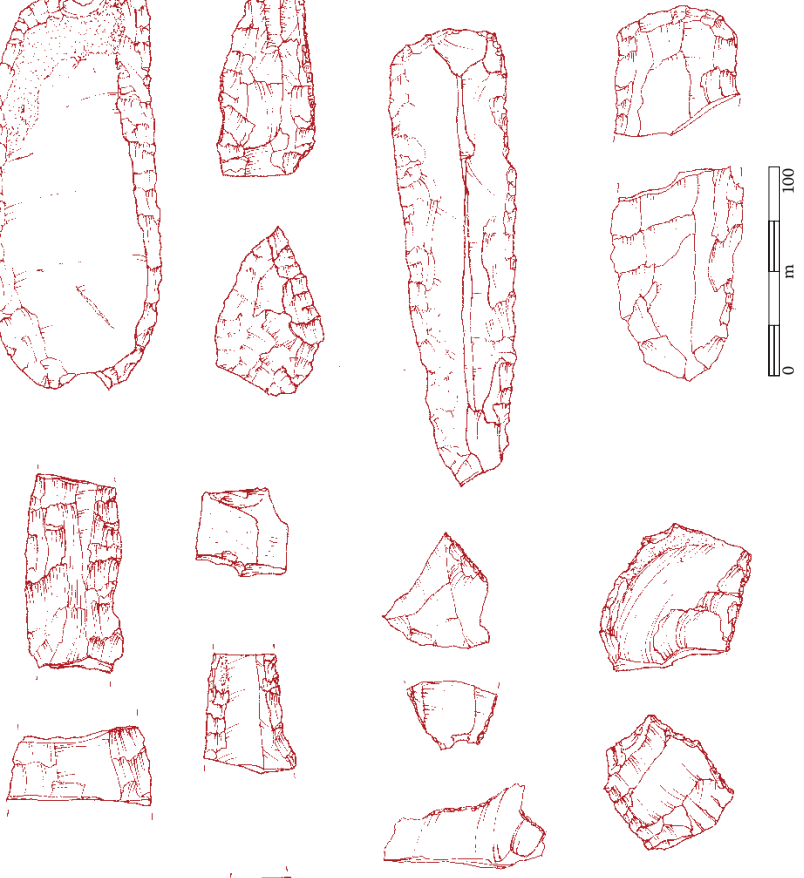

DER

(4)
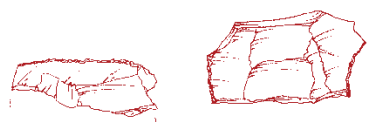